\title{
On the solution of differential equation system characterizing curve pair of constant Breadth by the Lucas collocation approximation
}

\author{
Muhammed Cetin, Huseyin Kocayigit and Mehmet Sezer \\ Department of Mathematics, Celal Bayar University, Manisa, Turkey \\ Received: 17 August 2015, Revised: 18 August 2015, Accepted: 30 September 2015 \\ Published online: 10 February 2016.
}

\begin{abstract}
In this study, for solving linear differential equation system characterizing curve pair of constant breadth according to Bishop frame in Euclidean 3-space, a new collocation method based on Lucas polynomials is introduced and hence the curve pair of constant breadth is determined. Furthermore, an error analysis based on residual error function is given for the method. To demonstrate the accuracy and effciency of the method, an example is given with the help of computer programmes Maple and Matlab.
\end{abstract}

Keywords: Curves pair of constant breadth, Bishop frame, Lucas polynomial and series, collocation points, system of differential equations.

\section{Introduction}

The curves of constant breadth were introduced by Euler in 1778 [1]. He investigated these curves in the plane. After him, many mathematicians were interested in these curves [2-11]. Mağden and Köse studied the curves of constant breadth in $E^{4}$-space in [12]. Then, the concepts related to constant breadth space curve were extended to $E^{n}$-space in [13]. Sezer established the differential equations characterizing curves of constant breadth and gave a criterion for these curves in [14]. Furthermore, Önder et al gave the differential equations characterizing the constant breadth timelike and spacelike curves in Minkowski 3-space $E_{1}^{3}$ in [15]. Also Kocayiğit and Önder showed that in $E_{1}^{3}$ spacelike and timelike curves of constant breadth are related to normal curves, spherical curves and helices in some special cases [16]. In [17], Kocayiğit and Çetin investigated the constant breadth space curves according to Bishop frame in Euclidean 3-space. Then, Çetin et al used a collocation method based on Taylor polynomials for the approximate solutions of the linear differential equation system characterizing constant breadth curves in Euclidean 3 -space $E^{3}$. So, curve pair of constant breadth is determined [18].

In [19], the collocation method based on Taylor polynomials was given by Sezer et al in order to find the approximate solutions of high-order systems of linear differential equations with variable coeffcients. In addition, Çetin et al presented an approximation method based on Lucas polynomials for the solution of the system of high-order linear differential equations with variable coeffcients under the mixed conditions [20].

In this study, we obtain the approximate solutions of the differential equation systems characterizing curve pair of 
constant breadth according to Bishop frame in Euclidean 3-space by using Lucas collocation method. Then, an example is given to demonstrate the effciency of the method.

\section{Preliminaries}

Firstly, we give some basic concepts on classical differential geometry of space curves. Let $\alpha(s)$ be an unit speed space curve and let $\{\vec{T}(s), \vec{N}(s), \vec{B}(s)\}$ be Frenet frame of this curve. The elements of the frame $\vec{T}, \vec{N}$ and $\vec{B}$ are called the unit tangent vector, the unit principal normal vector and the unit binormal vector of the curve, respectively. Moreover, $\kappa(s)$ and $\tau(s)$ are called curvature and torsion of the curve $\alpha$, respectively. The Frenet formulae are also well known as

$$
\left[\begin{array}{l}
\vec{T}^{\prime} \\
\overrightarrow{N^{\prime}} \\
\overrightarrow{B^{\prime}}
\end{array}\right]=\left[\begin{array}{ccc}
0 & \kappa & 0 \\
-\kappa & 0 & \tau \\
0 & -\tau & 0
\end{array}\right]\left[\begin{array}{l}
\vec{T} \\
\vec{N} \\
\vec{B}
\end{array}\right]
$$

where $\langle\vec{T}, \vec{T}\rangle=\langle\vec{N}, \vec{N}\rangle=\langle\vec{B}, \vec{B}\rangle=1$ and $\langle\vec{T}, \vec{N}\rangle=\langle\vec{N}, \vec{B}\rangle=\langle\vec{T}, \vec{B}\rangle=0$. The parallel transport frame is an alternative approach to defining a moving frame that is well-defined even when the curve has vanishing second derivative. We can parallel transport an orthonormal frame along a curve simply by paralel transporting each component of the frame [21].

Its mathematical properties derive from the observation that, while $\vec{T}(s)$ for a given curve model is unique, we may choose any convenient arbitrary basis $\left(\vec{N}_{1}(s), \vec{N}_{2}(s)\right)$ for the remainder of the frame, so long as it is in the normal plane perpendicular to $\vec{T}(s)$ at each point. If the derivatives of $\left(\vec{N}_{1}(s), \overrightarrow{N_{2}}(s)\right)$ depend only on $\vec{T}(s)$ and not each other, we can make $\overrightarrow{N_{1}}(s)$ and $\overrightarrow{N_{2}}(s)$ vary smoothly throughout the path regardless of the curvature. We may therefore choose the alternative frame equations

$$
\left[\begin{array}{l}
\vec{T}^{\prime} \\
\vec{N}_{1}^{\prime} \\
\vec{N}_{2}^{\prime}
\end{array}\right]=\left[\begin{array}{ccc}
0 & k_{1} & k_{2} \\
-k_{1} & 0 & 0 \\
-k_{2} & 0 & 0
\end{array}\right]\left[\begin{array}{l}
\vec{T} \\
\overrightarrow{N_{1}} \\
\overrightarrow{N_{2}}
\end{array}\right]
$$

where $\langle\vec{T}, \vec{T}\rangle=\left\langle\overrightarrow{N_{1}}, \overrightarrow{N_{1}}\right\rangle=\left\langle\overrightarrow{N_{2}}, \overrightarrow{N_{2}}\right\rangle=1$ and $\left\langle\vec{T}, \overrightarrow{N_{1}}\right\rangle=\left\langle\overrightarrow{N_{1}}, \overrightarrow{N_{2}}\right\rangle=\left\langle\vec{T}, \overrightarrow{N_{2}}\right\rangle=0[22,23]$

One can show that [22]

$$
\begin{gathered}
\kappa(s)=\sqrt{k_{1}^{2}+k_{2}^{2}}, \theta(s)=\arctan \left(\frac{k_{2}}{k_{1}}\right), \tau(s)=\frac{d \theta(s)}{d s} \\
k_{1}=\kappa \cos (\theta), k_{2}=\kappa \sin (\theta)
\end{gathered}
$$

and

$$
\vec{T}=\vec{T}, \vec{N}_{1}(s)=\vec{N} \cos (\theta)-\vec{B} \sin (\theta), \overrightarrow{N_{2}}(s)=\vec{N} \sin (\theta)+\vec{B} \cos (\theta)
$$

so that $k_{1}$ and $k_{2}$ effectively correspond to a Cartesian coordinate system for the polar coordinates $\kappa, \theta$ with $\theta=\int \tau(s) d s$. A fundamental ambiguity in the parallel transport frame compared to the Frenet frame thus arise from the arbitrary choice of an integration constant for $\theta_{0}$, which disappears $\tau$ from due to the differentiation [23]. 
It is well-known that the curvature $\kappa(s)$ of the curve (C) is defined by

$$
\lim _{\Delta s \rightarrow 0} \frac{\Delta \varphi}{\Delta s}=\frac{d \varphi}{d s}=\kappa(s)
$$

where $\varphi$ is the angle between the tangent $\vec{T}$ of the curve $\alpha$ and a given fixed direction at the point $\alpha(s)$.

\section{Lucas Collocation Method for Linear Differential Equation System with Variable Coefficients in Normal Form}

Çetin et al presented a collocation method based on Lucas polynomials to find the approximate solutions of high-order linear differential equation systems with variable coefficients in [20]. In this section, by means of the matrix relations between Lucas polynomials and their derivatives, we give a new method for solving the linear differential equation system in normal form as

$$
L\left[y_{i}(x)\right]=y_{i}^{\prime}(x)-\sum_{j=1}^{m} p_{i, j}(x) y_{j}(x)=g_{i}(x) \quad(i=1,2, \ldots, m) \quad(0 \leq a \leq x \leq b)
$$

under the initial conditions

$$
y_{i}(a)=c_{i}
$$

where $y_{i}(x)(i=1,2, \ldots, m)$ are unknown functions, $p_{i, j}(x)$ and $g_{i}(x)$ are the known continuous functions defined on interval $[a, b]$, and $c_{i}(i=1,2, \ldots, m)$ are the real constants. In this part, by means of Lucas collocation method with the help of the residual error function [24-27], we obtain the approximate solutions of the system (2) expressed in the truncated Lucas series

$$
y_{i, N, M}(x)=y_{i, N}(x)+e_{i, N, M}(x) \quad(i=1,2, \ldots, m)
$$

where

$$
y_{i}(x) \cong y_{i, N}(x)=\sum_{n=0}^{N} a_{i, n} L_{n}(x)
$$

is the Lucas polynomial solutions and

$$
e_{i, N, M}(x)=\sum_{n=0}^{M} a_{i, n}^{*} L_{n}(x) \quad(M>N)
$$

is the estimated error function obtained with the help of the residual error function. Here $a_{i, n}$ and $a_{i, n}^{*}$ are the unknown Lucas coefficients and $L_{n}(x)(n=0,1,2, \ldots, N)$ are the Lucas polynomials defined by [28,29],

$$
L_{0}(x)=2 ; L_{n}(x)=\sum_{k=0}^{\llbracket n / 2]} \frac{n}{n-k}\left(\begin{array}{c}
n-k \\
k
\end{array}\right) x^{n-2 k},(n \geq 1),[[n / 2]]=\left\{\begin{array}{c}
n / 2, n \text { even } \\
(n-1) / 2, n \text { odd }
\end{array} ;\right.
$$

In order to find the solutions of the system (2) under the initial conditions (3), we can use the collocation points defined by

$$
x_{k}=a+\frac{b-a}{N} k, k=0,1, \ldots, N, \quad 0 \leq a \leq x \leq b .
$$


On the other hand, we can write the approximate solutions $y_{i, N}(x)$ given by Eq.(4) in the matrix form

$$
y_{i, N}(x)=\mathrm{L}(x) \mathrm{A}_{i}, \quad(i=1,2, \ldots, m)
$$

where

$$
\mathrm{L}(x)=\left[L_{0}(x) L_{1}(x) L_{2}(x) \cdots L_{N}(x)\right]
$$

and

$$
\mathrm{A}_{i}=\left[\begin{array}{lllll}
a_{i, 0} & a_{i, 1} & a_{i, 2} & \cdots & a_{i, N}
\end{array}\right]^{T} .
$$

Here, the Lucas polynomials $L_{n}(x)$ can be written in the matrix form as

$$
\mathrm{L}(x)=\mathrm{X}(x) \mathrm{D}^{T}
$$

such that

$$
\mathrm{X}(x)=\left[\begin{array}{lllll}
1 & x & x^{2} & \cdots & x^{N}
\end{array}\right]
$$

and if $N$ is odd,

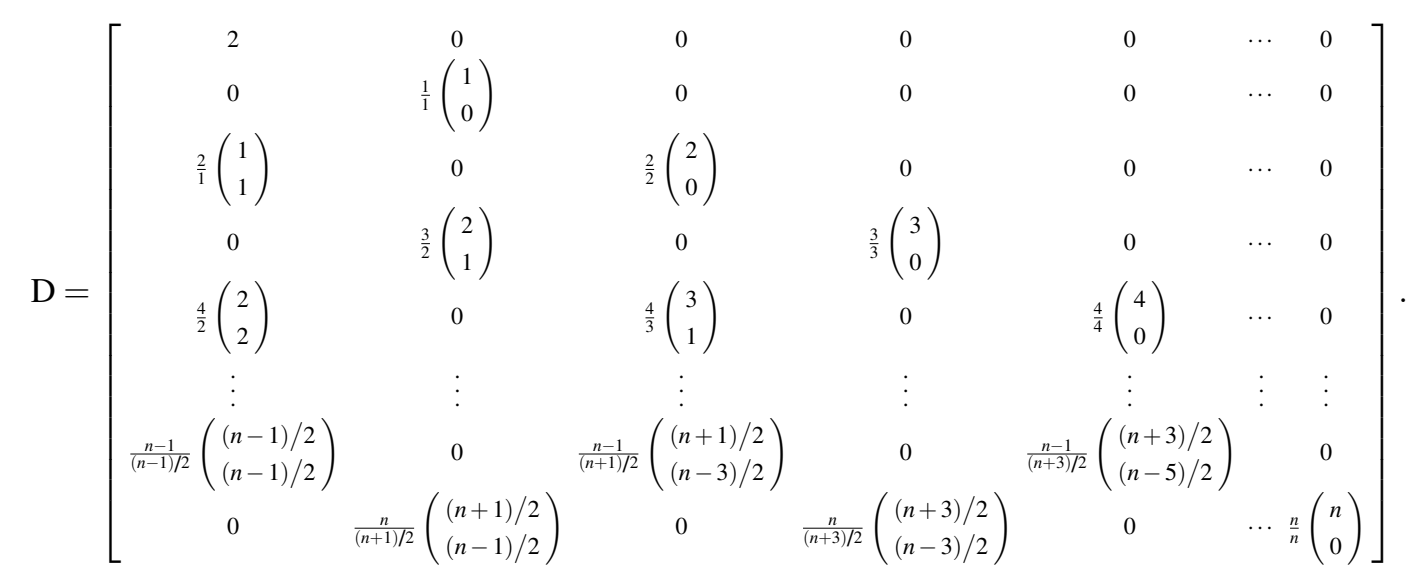

If $N$ is even,

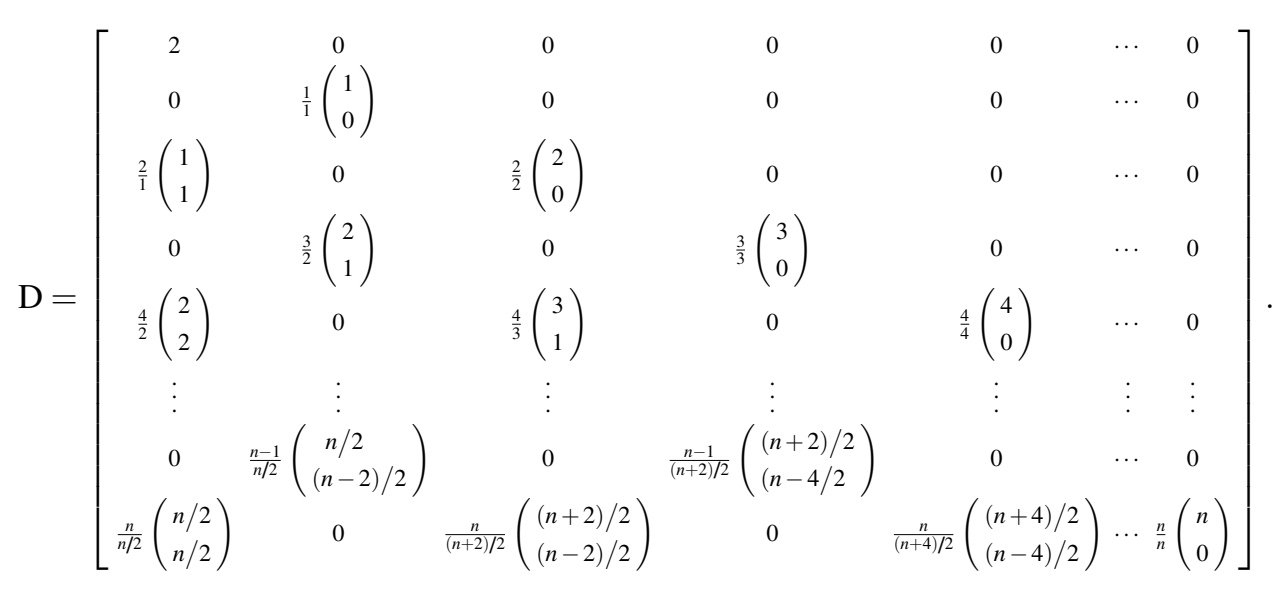


From Eq.(6) and Eq.(7), we obtain the matrix relation

$$
y_{i, N}(x)=\mathrm{X}(x) \mathrm{D}^{T} \mathrm{~A}_{i},(i=1,2, \ldots, m) .
$$

Therefore, the matrices $y_{i, N}(x),(i=1,2, \ldots, m)$ can be expressed as

$$
\mathrm{Y}(x)=\overline{\mathrm{X}}(x) \overline{\mathrm{D}} \mathrm{A} .
$$

where

$$
\begin{gathered}
\mathrm{Y}(x)=\left[\begin{array}{c}
y_{1, N}(x) \\
y_{2, N}(x) \\
\vdots \\
y_{m, N}(x)
\end{array}\right], \overline{\mathrm{X}}(x)=\left[\begin{array}{cccc}
\mathrm{X}(x) & 0 & \cdots & 0 \\
0 & \mathrm{X}(x) & \cdots & 0 \\
\vdots & \vdots & \ddots & \vdots \\
0 & 0 & \cdots & \mathrm{X}(x)
\end{array}\right], \\
\overline{\mathrm{D}}=\left[\begin{array}{cccc}
\mathrm{D}^{T} & 0 & \cdots & 0 \\
0 & \mathrm{D}^{T} & \cdots & 0 \\
\vdots & \vdots & \ddots & \vdots \\
0 & 0 & \cdots & \mathrm{D}^{T}
\end{array}\right], \mathrm{A}=\left[\begin{array}{c}
\mathrm{A}_{1} \\
\mathrm{~A}_{2} \\
\vdots \\
\mathrm{A}_{m}
\end{array}\right]
\end{gathered}
$$

Also, the relation between the matrix $\mathrm{X}(x)$ and its derivative $\mathrm{X}^{\prime}(x)$ is

$$
\mathrm{X}^{\prime}(x)=\mathrm{X}(x) \mathrm{B}
$$

where

$$
\mathrm{B}=\left[\begin{array}{ccccc}
0 & 1 & 0 & \cdots & 0 \\
0 & 0 & 2 & \cdots & 0 \\
\vdots & \vdots & \vdots & \ddots & \vdots \\
0 & 0 & 0 & \cdots & N \\
0 & 0 & 0 & \cdots & 0
\end{array}\right]
$$

By using the relations (8) and (10), we obtain the following matrix relation

$$
y_{i, N}^{\prime}(x)=\mathrm{X}(x) \mathrm{BD}^{T} \mathrm{~A}_{i} . \quad(i=1,2, \ldots, m)
$$

Hence, we can write the matrix relations as follow

$$
\mathrm{Y}^{\prime}(x)=\overline{\mathrm{X}}(x) \overline{\mathrm{BD}} \mathrm{A}
$$

where

$$
\mathrm{Y}^{\prime}(x)=\left[\begin{array}{c}
y_{1, N}^{\prime}(x) \\
y_{2, N}^{\prime}(x) \\
\vdots \\
y^{\prime}{ }_{m, N}(x)
\end{array}\right], \overline{\mathrm{B}}=\left[\begin{array}{cccc}
\mathrm{B} & 0 & \cdots & 0 \\
0 & \mathrm{~B} & \cdots & 0 \\
\vdots & \vdots & \ddots & \vdots \\
0 & 0 & \cdots & \mathrm{B}
\end{array}\right] .
$$




\section{Method for solution}

We can write the system (2) in the matrix form

$$
\mathrm{Y}^{\prime}(x)=\mathrm{P}(x) \mathrm{Y}(x)+\mathrm{G}(x)
$$

where

$$
\mathrm{P}(x)=\left[\begin{array}{cccc}
p_{1,1}(x) & p_{1,2}(x) & \cdots & p_{1, m}(x) \\
p_{2,1}(x) & p_{2,1}(x) & \cdots & p_{2,1}(x) \\
\vdots & \vdots & \ddots & \vdots \\
p_{m, 1}(x) & p_{m, 2}(x) & \cdots & p_{m, m}(x)
\end{array}\right], \mathrm{G}(x)=\left[\begin{array}{c}
g_{1}(x) \\
g_{2}(x) \\
\vdots \\
g_{m}(x)
\end{array}\right] .
$$

By using the collocation points given by (5) into Eq.(13), we obtain the system of matrix equations

$$
\mathrm{Y}^{\prime}\left(x_{k}\right)=\mathrm{P}\left(x_{k}\right) \mathrm{Y}\left(x_{k}\right)+\mathrm{G}\left(x_{k}\right),(k=0,1, \ldots, N) \text {. }
$$

Briefly, the fundamental matrix equation is

$$
\mathrm{Y}^{\prime}=\mathrm{PY}+\mathrm{G}
$$

where

$$
\mathrm{P}=\left[\begin{array}{cccc}
\mathrm{P}\left(x_{0}\right) & 0 & \cdots & 0 \\
0 & \mathrm{P}\left(x_{1}\right) & \cdots & 0 \\
\vdots & \vdots & \ddots & \vdots \\
0 & 0 & \cdots & \mathrm{P}\left(x_{N}\right)
\end{array}\right], \mathrm{Y}=\left[\begin{array}{c}
\mathrm{Y}\left(x_{0}\right) \\
\mathrm{Y}\left(x_{1}\right) \\
\vdots \\
\mathrm{Y}\left(x_{N}\right)
\end{array}\right], \mathrm{Y}^{\prime}=\left[\begin{array}{c}
\mathrm{Y}^{\prime}\left(x_{0}\right) \\
\mathrm{Y}^{\prime}\left(x_{1}\right) \\
\vdots \\
\mathrm{Y}^{\prime}\left(x_{N}\right)
\end{array}\right], \mathrm{G}=\left[\begin{array}{c}
\mathrm{G}\left(x_{0}\right) \\
\mathrm{G}\left(x_{1}\right) \\
\vdots \\
\mathrm{G}\left(x_{N}\right)
\end{array}\right]
$$

From the relations (9) and (12) and the collocation points given by (5), we obtain

$$
\mathrm{Y}\left(x_{k}\right)=\overline{\mathrm{X}}\left(x_{k}\right) \overline{\mathrm{D}} \mathrm{A} \text { and } \mathrm{Y}^{\prime}\left(x_{k}\right)=\overline{\mathrm{X}}\left(x_{k}\right) \overline{\mathrm{BDA}},(k=0,1, \ldots, N)
$$

or briefly

$$
\mathrm{Y}=\mathrm{X} \overline{\mathrm{D}} \mathrm{A} \text { and } \mathrm{Y}^{\prime}=\mathrm{X} \overline{\mathrm{BD}} \mathrm{A}
$$

where

$$
\mathrm{X}=\left[\begin{array}{c}
\overline{\mathrm{X}}\left(x_{0}\right) \\
\overline{\mathrm{X}}\left(x_{1}\right) \\
\vdots \\
\overline{\mathrm{X}}\left(x_{N}\right)
\end{array}\right], \overline{\mathrm{X}}\left(x_{k}\right)=\left[\begin{array}{cccc}
\mathrm{X}\left(x_{k}\right) & 0 & \cdots & 0 \\
0 & \mathrm{X}\left(x_{k}\right) & \cdots & 0 \\
\vdots & \vdots & \ddots & \vdots \\
0 & 0 & \cdots & \mathrm{X}\left(x_{k}\right)
\end{array}\right]
$$

By substituting the relations given by (15) into Eq.(14), we gain the fundamental matrix equation as

$$
\{\mathrm{X} \overline{\mathrm{BD}}-\mathrm{PX} \overline{\mathrm{D}}\} \mathrm{A}=\mathrm{G}
$$

In Eq.(16) the full dimensions of the matrices $\mathrm{P}, \mathrm{X}, \overline{\mathrm{B}}, \overline{\mathrm{D}}, \mathrm{A}$ and $\mathrm{G}$ are $m(N+1) \times m(N+1), m(N+1) \times m(N+1)$, $m(N+1) \times m(N+1), m(N+1) \times m(N+1), m(N+1) \times 1$ and $m(N+1) \times 1$, respectively.

The fundamental matrix equation (16) corresponding to Eq.(2) can be written in the form

$$
\mathrm{WA}=\mathrm{G} \text { or }[\mathrm{W} ; \mathrm{G}] \text {. }
$$


This is a linear system of $m(N+1)$ algebraic equations in $m(N+1)$ the unknown Lucas coefficients such that

$$
\mathrm{W}=\mathrm{X} \overline{\mathrm{BD}}-\mathrm{PX} \overline{\mathrm{D}}=\left[w_{p, q}\right], \quad p, q=1,2, \ldots, m(N+1) .
$$

By using the conditions given by (5) and the relations (9), the matrix form for the conditions is obtained as

$$
\overline{\mathrm{X}}(a) \overline{\mathrm{D}} \mathrm{A}=\mathrm{C}
$$

where

$$
\mathrm{C}=\left[\begin{array}{llll}
c_{1} & c_{2} & \cdots & c_{m}
\end{array}\right]^{T}
$$

Hence, the fundamental matrix form for conditions is

$$
\mathrm{UA}=\mathrm{C} \text { or }[\mathrm{U} ; \mathrm{C}]
$$

such that

$$
\mathrm{U}=\overline{\mathrm{X}}(a) \overline{\mathrm{D}}
$$

Consequently, we obtain the Lucas polynomial solution of the system (2) under the initial conditions (3) by replacing the row matrices (19) by last rows of the matrix (17). Then, we obtain the new augmented matrix

$$
\widetilde{\mathrm{W}} \mathrm{A}=\widetilde{\mathrm{G}} \text { or }[\widetilde{\mathrm{W}} ; \widetilde{\mathrm{G}}]
$$

If $\operatorname{rank} \widetilde{\mathrm{W}}=\operatorname{rank}[\widetilde{\mathrm{W}} ; \widetilde{\mathrm{G}}]=N+1$, then we can write

$$
\mathrm{A}=(\widetilde{\mathrm{W}})^{-1} \widetilde{\mathrm{G}}
$$

By solving this linear system, the unknown Lucas coefficients matrix A is determined and $a_{i, 0}, a_{i, 1}, \ldots, a_{i, N}(i=1,2, \ldots, m)$ are substituted in Eq.(4). Thus, we find the Lucas polynomial solutions

$$
y_{i, N}(x)=\sum_{n=0}^{N} a_{i, n} L_{n}(x), \quad(i=1,2, \ldots, m) .
$$

\section{Residual correction and error estimation}

In this section, we give an error estimation for the Lucas polynomial solutions (4) with the residual error function [24-27]. In addition, we develop the Lucas polynomial solutions (4) by means of the residual error function. Firstly, we can define the residual error function for the Lucas collocation method as

$$
R_{i, N}(x)=L\left[y_{i, N}(x)\right]-g_{i}(x), \quad(i=1,2, \ldots, m) .
$$

Here, $y_{i, N}(x)$ represent the Lucas polynomial solutions given by (4) of the problem (2) and (3). Hence, $y_{j, N}(x)$ satisfies the problem

$$
\left\{\begin{array}{l}
y_{i, N}^{\prime}(x)-\sum_{j=1}^{m} p_{i, j}(x) y_{j, N}(x)=g_{i}(x)+R_{i, N}(x), \quad(i=1,2, \ldots, m) \\
y_{i, N}(a)=c_{i}, \quad(i=1,2, \ldots, m)
\end{array}\right.
$$


Also, the error function $e_{i, N}(x)$ can be defined as

$$
e_{i, N}(x)=y_{i}(x)-y_{i, N}(x)
$$

where $y_{i}(x)$ are the exact solutions of the problem (2) and (3). From Eqs.(2), (3), (22) and (23), we obtain a system of error differential equations

$$
L\left[e_{i, N}(x)\right]=L\left[y_{i}(x)\right]-L\left[y_{i, N}(x)\right]=-R_{i, N}(x)
$$

with the homogeneous initial conditions

$$
e_{i, N}(a)=0,(i=1,2, \ldots, m)
$$

or openly, the error problem can be expressed as

$$
\left\{\begin{array}{l}
e^{\prime}{ }_{i, N}(x)-\sum_{j=1}^{m} p_{i, j}(x) e_{j, N}(x)=-R_{i, N}(x), \quad(i=1,2, \ldots, m) \\
e_{i, N}(a)=0, \quad(i=1,2, \ldots, m) .
\end{array}\right.
$$

Here, the nonhomegeneous initial conditions

$$
y_{i}(a)=c_{i} \text { and } y_{i, N}(a)=c_{i}
$$

are reduced to homogeneous initial conditions

$$
e_{i, N}(a)=0 .
$$

The error problem (24) can be solved by using the prosedure given in Section 3-4. Thus, we obtain the approximation

$$
e_{i, N, M}(x)=\sum_{n=0}^{M} a_{i, n}^{*} L_{n}(x), \quad(M>N, \quad i=1,2, \ldots, m)
$$

to $e_{i, N}(x)$. Consequently, the corrected Lucas polynomial solution $y_{i, N, M}(x)=y_{i, N}(x)+e_{i, N, M}(x)$ is obtained by means of the polynomials $y_{i, N}(x)$ and $e_{i, N, M}(x)$. In addition, we construct the error function $e_{i, N}(x)=y_{i}(x)-y_{i, N}(x)$, the estimated error function $e_{i, N, M}(x)$ and the corrected error function $E_{i, N, M}(x)=y_{i}(x)-y_{i, N, M}(x)$.

\section{Illustration}

In this section, we give an example. In tables and figures, we calculate the values of the Lucas polynomial solutions $y_{i, N}(x)$, the corrected Lucas polynomial solutions $y_{i, N, M}(x)=y_{i, N}(x)+e_{i, N, M}(x)$ and the estimated absolute error function $\left|e_{i, N, M}(x)\right|$.

Definition 1. A pair of space curves $(C)$ and $\left(C^{*}\right)$ in $E^{3}$ for which the tangents at the corresponding points $\alpha(s)$ and $\alpha^{*}\left(s^{*}\right)$ are parallel and in opposite directions, and the distance between these points is always constant are called space curve pair of constant breadth [11].

Let $(C)$ and $\left(C^{*}\right)$ be a pair of unit-speed curves in Euclidean 3-space with non-zero Bishop curvatures and let those curves have paralel tangents in opposite directions at the corresponding points $\alpha(s)$ and $\alpha^{*}\left(s^{*}\right)$, repectively. Hence, the position vector of the curve $\left(C^{*}\right)$ at the point $\alpha^{*}\left(s^{*}\right)$ can be written as

$$
\overrightarrow{\alpha^{*}}\left(s^{*}\right)=\vec{\alpha}(s)+\lambda_{1}(s) \vec{T}(s)+\lambda_{2}(s) \overrightarrow{N_{1}}(s)+\lambda_{3}(s) \overrightarrow{N_{2}}(s)
$$


where $\lambda_{i}(s)(i=1,2,3)$ are differentiable functions of $s$ which is arc lenght of $(C)$. Denote by $\left\{\vec{T}, \overrightarrow{N_{1}}, \overrightarrow{N_{2}}\right\}, k_{1}$ and $k_{2}$ the moving Bishop frame, Bishop curvatures along the curve $(C)$, respectively. And denote by $\left\{\overrightarrow{T^{*}}, \overrightarrow{N_{1}^{*}}, \overrightarrow{N_{2}^{*}}\right\}, k_{1}^{*}$ and $k_{2}^{*}$ the moving Bishop frame, Bishop curvatures along the curve $\left(C^{*}\right)$, respectively.

Theorem 1. The general differential equations system characterizing space curve pair of constant breadth according to Bishop frame in $E^{3}$ is as follows [17].

$$
\left\{\begin{array}{l}
\frac{d \lambda_{1}}{d s}=k_{1} \lambda_{2}+k_{2} \lambda_{3} \\
\frac{d \lambda_{2}}{d s}=-k_{1} \lambda_{1} \\
\frac{d \lambda_{3}}{d s}=-k_{2} \lambda_{1}
\end{array}\right.
$$

where $k_{1}$ and $k_{2}$ are Bishop curvatures defined by

$$
k_{1}=\kappa(s) \cos (\theta) \text { and } k_{1}=\kappa(s) \sin (\theta),\left(\theta=\int \tau(s) d s\right) .
$$

The position vector of the curve $\left(C^{*}\right)$ at the point $\alpha^{*}\left(s^{*}\right)$ in terms of $\alpha^{\prime}, \alpha^{\prime \prime}, \alpha^{\prime \prime \prime}$ and $k_{1}, k_{2}$ by means of the Bishop formulae as follows [18].

$$
\overrightarrow{\alpha^{*}}\left(s^{*}\right)=\left(\frac{k_{1} \lambda_{3}-k_{2} \lambda_{2}}{\mu}\right) \vec{\alpha}^{\prime \prime \prime}+\left(\frac{k_{2}{ }^{\prime} \lambda_{2}-k_{1}{ }^{\prime} \lambda_{3}}{\mu}\right) \vec{\alpha}^{\prime \prime}+\left[\frac{\left(k_{1}^{2}+k_{2}^{2}\right)\left(k_{1} \lambda_{3}-k_{2} \lambda_{2}\right)}{\mu}+\lambda_{1}\right] \vec{\alpha}^{\prime}+\vec{\alpha}
$$

such that

$$
\mu=k_{1}^{2}\left(\frac{k_{2}}{k_{1}}\right)^{\prime} .
$$

Example 1. Let us consider the curve $\alpha:[0,2 \pi] \rightarrow E^{3}$ given by

$$
\alpha(s)=\left(\cos \left(\frac{s}{2}\right), \sin \left(\frac{s}{2}\right), \frac{\sqrt{3} s}{2}\right) .
$$

For the curve $\alpha$ curvature, torsion and Bishop elements are calculated as

$$
\begin{gathered}
\kappa=\frac{1}{4}, \tau=\frac{\sqrt{3}}{4}, \theta=\frac{\sqrt{3} s}{4} \\
k_{1}=\frac{1}{4} \cos \left(\frac{\sqrt{3} s}{4}\right) \text { and } k_{2}=\frac{1}{4} \sin \left(\frac{\sqrt{3} s}{4}\right) .
\end{gathered}
$$

Substituting (27) in (26), we obtain

$$
\left\{\begin{array}{l}
\lambda_{1}{ }^{\prime}=\frac{1}{4} \cos \left(\frac{\sqrt{3} s}{4}\right) \lambda_{2}+\frac{1}{4} \sin \left(\frac{\sqrt{3} s}{4}\right) \lambda_{3} \\
\lambda_{2}{ }^{\prime}=-\frac{1}{4} \cos \left(\frac{\sqrt{3} s}{4}\right) \lambda_{1} \\
\lambda_{3}{ }^{\prime}=-\frac{1}{4} \sin \left(\frac{\sqrt{3} s}{4}\right) \lambda_{1}
\end{array}\right.
$$

We can find the approximate solutions of the problem (28) by using Lucas collocation method above mentioned. We suppose that the initial conditions for $\lambda_{1}(s), \lambda_{2}(s)$ and $\lambda_{3}(s)$ as follows

$$
\lambda_{1}(0)=1, \lambda_{2}(0)=4, \lambda_{3}(0)=2 .
$$

The approximate solutions $\lambda_{1,3}(s), \lambda_{2,3}(s)$ and $\lambda_{3,3}(s)$ by the truncated Lucas series for $N=3$ are given by

$$
\lambda_{i, 3}(s)=\sum_{n=0}^{3} a_{i, n} s^{n}, \quad(i=1,2,3) .
$$


The set of the collocation points for $a=0, b=2 \pi$ and $N=3$ is calculated as

$$
\left\{s_{0}=0, s_{1}=\frac{2 \pi}{3}, s_{2}=\frac{4 \pi}{3}, s_{3}=2 \pi\right\} .
$$

We can write the fundamental matrix equation of the problem (28) from Eq.(16) as

$$
\{\mathrm{S} \overline{\mathrm{BD}}-\mathrm{PS} \overline{\mathrm{D}}\} \mathrm{A}=\mathrm{G}
$$

By using the method in Section 3-4, the approximate solutions of the problem (28) for $N=3$ are obtaied as

$$
\begin{aligned}
\lambda_{1,3}= & 1.0000000000000000278+0.9999999999999994904 s+(0.230862972284423679 e-1) s^{2} \\
& -(0.268502435025769032 e-1) s^{3} \\
\lambda_{2,3}= & 4.000000000000000318-0.2499999999999998461 s-0.153376809980715401 s^{2} \\
& +(0.332975802130747873 e-1) s^{3} \\
\lambda_{3,3}= & 2.000000000000000098+(0.36 e-17) s-0.172375652146830871 s^{2}+(0.107444813448467590 e-1) s^{3} .
\end{aligned}
$$

In order to calculate the corrected Lucas polynomial solutions, let us consider the error problem

$$
\left\{\begin{array}{l}
e^{\prime}{ }_{1,3}(s)-\frac{1}{4} \cos \left(\frac{\sqrt{3} s}{4}\right) e_{2,3}(s)-\frac{1}{4} \sin \left(\frac{\sqrt{3} s}{4}\right) e_{3,3}(s)=-R_{1,3}(s) \\
e^{\prime}{ }_{2,3}(s)+\frac{1}{4} \cos \left(\frac{\sqrt{3} s}{4}\right) e_{1,3}(s)=-R_{2,3}(s) \\
e^{\prime}{ }_{3,3}(s)+\frac{1}{4} \sin \left(\frac{\sqrt{3} s}{4}\right) e_{1,3}(s)=-R_{3,3}(s)
\end{array}\right.
$$

such that $e_{1,3}(0)=0, e_{2,3}(0)=0, e_{3,3}(0)=0$ and the residual functions are

$$
\left\{\begin{array}{l}
R_{1,3}(s)=\lambda_{1,3}^{\prime}(s)-\frac{1}{4} \cos \left(\frac{\sqrt{3} s}{4}\right) \lambda_{2,3}(s)-\frac{1}{4} \sin \left(\frac{\sqrt{3} s}{4}\right) \lambda_{3,3}(s) \\
R_{2,3}(s)=\lambda_{2,3}^{\prime}(s)+\frac{1}{4} \cos \left(\frac{\sqrt{3} s}{4}\right) \lambda_{1,3}(s) \\
R_{3,3}(s)=\lambda_{3,3}^{\prime}(s)+\frac{1}{4} \sin \left(\frac{\sqrt{3} s}{4}\right) \lambda_{1,3}(s) .
\end{array}\right.
$$

By solving the error problem (29) for $M=4$, the estimeted Lucas error function approximations $e_{1,3,4}(s), e_{2,3,4}(s)$ and $e_{3,3,4}(s)$ to $e_{1,3}(s), e_{2,3}(s)$ and $e_{3,3}(s)$ are obtained as

$$
\begin{aligned}
e_{1,3,4}(s)= & -(0.156824 e-14)+(0.11693 e-14) s+(0.6879998068480421702 e-1) s^{2} \\
& -(0.310172009517819015 e-1) s^{3}+(0.358834023138630138 e-2) s^{4} \\
e_{2,3,4}(s)= & -(0.39300 e-15)-(0.1874 e-15) s-(0.5090722047025802480 e-1) s^{2} \\
& +(0.259317019347674524 e-1) s^{3}-(0.329254069115442410 e-2) s^{4} \\
e_{3,3,4}(s)= & -(0.2660 e-16)+(0.4 e-18) s+0.12141208040163570600 s^{2} \\
& -(0.587889512477086652 e-1) s^{3}+(0.694771346566892910 e-2) s^{4}
\end{aligned}
$$

Hence, we can calculate the corrected Lucas polynomial solutions for $M=4$ as

$$
\lambda_{1,3,4}(s)=1+s+(0.9188627791 e-1) s^{2}-(0.5786744445 e-1) s^{3}+(0.358834023138630138 e-2) s^{4}
$$




$$
\begin{aligned}
\lambda_{2,3,4}(s)= & 4-0.25 s-0.2042840305 s^{2}+(0.5922928214 e-1) s^{3}-(0.329254069115442410 e-2) s^{4} \\
\lambda_{3,3,4}(s)= & 2+(0.40 e-17) s-(0.509635717 e-1) s^{2}-(0.4804446991 e-1) s^{3} \\
& +(0.694771346566892910 e-2) s^{4} .
\end{aligned}
$$

Similarly, we can calculate corrected Lucas polynomial solutions of the problem for different values of $M$ as follows.

For $M=6$, the approximate solutions are

$$
\begin{aligned}
\lambda_{1,3,6}(s)= & +s+(0.7722888773 e-1) s^{2}-(0.4104315343 e-1) s^{3}-(0.22724979443498158924 e-2) s^{4} \\
& +(0.780946093192976150 e-3) s^{5}-(0.328376246375317054 e-4) s^{6} \\
\lambda_{2,3,6}(s)= & -0.25 s-0.1103232354 s^{2}-(0.1994160108 e-1) s^{3}+(0.20829016919357967950 e-1) s^{4} \\
& -(0.300128817280402279 e-2) s^{5}+(0.121540192683128125 e-3) s^{6} \\
\lambda_{3,3,6}(s)= & +(0.21200 e-15) s-(0.506308036 e-1) s^{2}-(0.3981039691 e-1) s^{3} \\
& -(0.453694473233072774 e-3) s^{4}+(0.205140773861414456 e-2) s^{5} \\
& -(0.179925190950981802 e-3) s^{6} .
\end{aligned}
$$

For $M=8$, the approximate solutions are

$$
\begin{aligned}
\lambda_{1,3,8}(s)= & +s+(0.7692606583 e-1) s^{2}-(0.4159234923 e-1) s^{3}-(0.449362456550112302 e-5) s^{7} \\
& +(0.139070836974095252 e-6) s^{8}-(0.16320200368388819474 e-2) s^{4} \\
& +(0.51987164532193863786 e-3) s^{5}+(0.17566163126409611616 e-4) s^{6} \\
\lambda_{2,3,8}(s)= & -0.25 s-0.1263557000 s^{2}+(0.440484041 e-2) s^{3}+(0.702607695411888776 e-4) s^{7} \\
& -(0.221094199053020487 e-5) s^{8}+(0.55555395786277287546 e-2) s^{4} \\
& +(0.19018036742970799332 e-2) s^{5}-(0.71348969074471050696 e-3) s^{6} \\
\lambda_{3,3,8}(s)= & -(0.10153678 e-14) s-(0.547180547 e-1) s^{2} \\
& -(0.3488333727 e-1) s^{3}-(0.394613814952812954 e-4) s^{7} \\
& +(0.262930012001328484 e-5) s^{8}-(0.22358445035079499478 e-2) s^{4} \\
& +(0.19778385702964519922 e-2) s^{5}+(0.471203000543214462 e-5) s^{6} .
\end{aligned}
$$

Table 1: Comparison of the approximate solutions $\lambda_{1, N, M}(s)$ for $N=3$ and $M=4,6,8$.

\begin{tabular}{c|c|c|c}
\hline$s_{i}$ & $\lambda_{1,3,4}\left(s_{i}\right)$ & $\lambda_{1,3,6}\left(s_{i}\right)$ & $\lambda_{1,3,8}\left(s_{i}\right)$ \\
\hline 0 & 1 & 1 & 1 \\
$\pi / 4$ & 1.815408399 & 1.812513502 & 1.812237341 \\
$2 \pi / 4$ & 2.595080941 & 2.595416426 & 2.594599540 \\
$3 \pi / 4$ & 3.219959948 & 3.229119453 & 3.227980131 \\
$4 \pi / 4$ & 3.603756833 & 3.617269033 & 3.615951406 \\
$5 \pi / 4$ & 3.692952101 & 3.700880003 & 3.699449695 \\
$6 \pi / 4$ & 3.466795343 & 3.466918839 & 3.465760519 \\
$7 \pi / 4$ & 2.937305243 & 2.951337540 & 2.950470076 \\
$2 \pi$ & 2.149269576 & 2.236558148 & 2.231861391 \\
\hline
\end{tabular}


Table 2: Comparison of the approximate solutions $\lambda_{2, N, M}(s)$ for $N=3$ and $M=4,6,8$.

\begin{tabular}{c|c|c|c}
\hline$s_{i}$ & $\lambda_{2,3,4}\left(s_{i}\right)$ & $\lambda_{2,3,6}\left(s_{i}\right)$ & $\lambda_{2,3,8}\left(s_{i}\right)$ \\
\hline 0 & 4 & 4 & 4 \\
$\pi / 4$ & 3.705079965 & 3.732993502 & 3.730369359 \\
$2 \pi / 4$ & 3.312765003 & 3.357732448 & 3.355470705 \\
$3 \pi / 4$ & 2.950123340 & 2.982434532 & 2.980565716 \\
$4 \pi / 4$ & 2.714155384 & 2.734770119 & 2.732536442 \\
$5 \pi / 4$ & 2.671793724 & 2.705579966 & 2.703910773 \\
$6 \pi / 4$ & 2.859903133 & 2.913132538 & 2.910841823 \\
$7 \pi / 4$ & 3.285280563 & 3.287920907 & 3.285146483 \\
$2 \pi$ & 3.924655149 & 3.677999237 & 3.707601606 \\
\hline
\end{tabular}

Table 3: Comparison of the approximate solutions $\lambda_{3, N, M}(s)$ for $N=3$ and $M=4,6,8$.

\begin{tabular}{c|c|c|c}
\hline$s_{i}$ & $\lambda_{3,3,4}\left(s_{i}\right)$ & $\lambda_{3,3,6}\left(s_{i}\right)$ & $\lambda_{3,3,8}\left(s_{i}\right)$ \\
\hline 0 & 2 & 2 & 2 \\
$\pi / 4$ & 1.947930489 & 1.949879504 & 1.949081651 \\
$2 \pi / 4$ & 1.730340565 & 1.734929919 & 1.734327739 \\
$3 \pi / 4$ & 1.302743559 & 1.302368008 & 1.302037820 \\
$4 \pi / 4$ & 0.684100035 & 0.676520866 & 0.676111342 \\
$5 \pi / 4$ & -0.043182214 & -0.043622926 & -0.043278513 \\
$6 \pi / 4$ & -0.733248164 & -0.717268464 & -0.717296996 \\
$7 \pi / 4$ & -1.176795563 & -1.225075999 & -1.225287853 \\
$2 \pi$ & -1.101074493 & -1.562828725 & -1.508434184 \\
\hline
\end{tabular}

It is seen from Table 1-3 that the approximate solutions are almost identical. We can write the distance function $d_{N, M}$ from (25) as

$$
d_{N, M}=\sqrt{\lambda_{1, N, M}^{2}+\lambda_{2, N, M}^{2}+\lambda_{3, N, M}^{2}}=k, k \in .
$$

Now, let us calculate the values of $d_{N, M}$ for $N=3$ and $M=4,6,8$. Hence,

$$
\begin{aligned}
d_{3,4}= & (0.5 e-9)\left[-(0.236741589992000 e 19) s^{2}+(0.180734423860000 e 19) s^{3}+287950928880522 s^{8}\right. \\
& -(0.844546189650672 e 17) s^{5}+(0.418460996545252 e 17) s^{6}-(0.589169688884716 e 16) s^{7} \\
& \left.+(0.84 e 20)-(0.335799929391549 e 18) s^{4}\right]^{(1 / 2)} \\
d_{3,6}= & (0.1 e-10)\left[(0.131848677860000 e 22) s^{2}-(0.191241309980000 e 22) s^{3}+(0.21 e 24)\right. \\
& -(0.159390765639094 e 19) s^{7}+(0.317809394084149 e 19) s^{8}-(0.118264691963043 e 19) s^{9} \\
& +(0.192015156647224 e 18) s^{10}-(0.151904297315817 e 17) s^{11}+482234023680346 s^{12} \\
& \left.+(0.108855850895550 e 22) s^{4}-(0.271104120918496 e 21) s^{5}+(0.201564518124391 e 20) s^{6}\right]^{(1 / 2)} \\
d_{3,8}= & (0.2 e-12)\left[101536780000 s-(0.334142178500000 e 24) s^{2}+(0.738766435 e 24) s^{3}+(0.525 e 27)\right. \\
& -(0.255765080554550 e 21) s^{9}+(0.957793165321548 e 20) s^{10}-(0.479903869336240 e 20) s^{11} \\
& +(0.242465055651551 e 18) s^{14}-(0.129861616448192 e 17) s^{15}+(0.144774173512770 e 20) s^{12} \\
& -(0.246636805567016 e 19) s^{13}+295520607607205 s^{16}+(0.127687136841916 e 23) s^{7} \\
& +(0.138307097481750 e 21) s^{8}-(0.706819302174100 e 24) s^{4}+(0.360724721009030 e 24) s^{5} \\
& \left.-(0.100231952403750 e 24) s^{6}\right]^{(1 / 2)} .
\end{aligned}
$$


Table 4: Numerical results of distance functions $d_{N, M}$ for $N=3$ and $M=4,6,8$.

\begin{tabular}{c|c|c|c}
\hline$s_{i}$ & $d_{3,4}\left(s_{i}\right)$ & $d_{3,6}\left(s_{i}\right)$ & $d_{3,8}\left(s_{i}\right)$ \\
\hline 0 & 4.582575 & 4.582576 & 4.582576 \\
$\pi / 4$ & 4.562648 & 4.585027 & 4.582442 \\
$2 \pi / 4$ & 4.550048 & 4.584816 & 4.582469 \\
$3 \pi / 4$ & 4.557248 & 4.584571 & 4.582459 \\
$4 \pi / 4$ & 4.563080 & 4.584897 & 4.582465 \\
$5 \pi / 4$ & 4.558316 & 4.584602 & 4.582459 \\
$6 \pi / 4$ & 4.553611 & 4.584795 & 4.582468 \\
$7 \pi / 4$ & 4.561324 & 4.584935 & 4.582444 \\
$2 \pi$ & 4.608106 & 4.579553 & 4.582891 \\
\hline
\end{tabular}

Now, let us draw the graphics of the distance functions $d_{N, M}$ for $N=3$ and $M=4,6,8$.

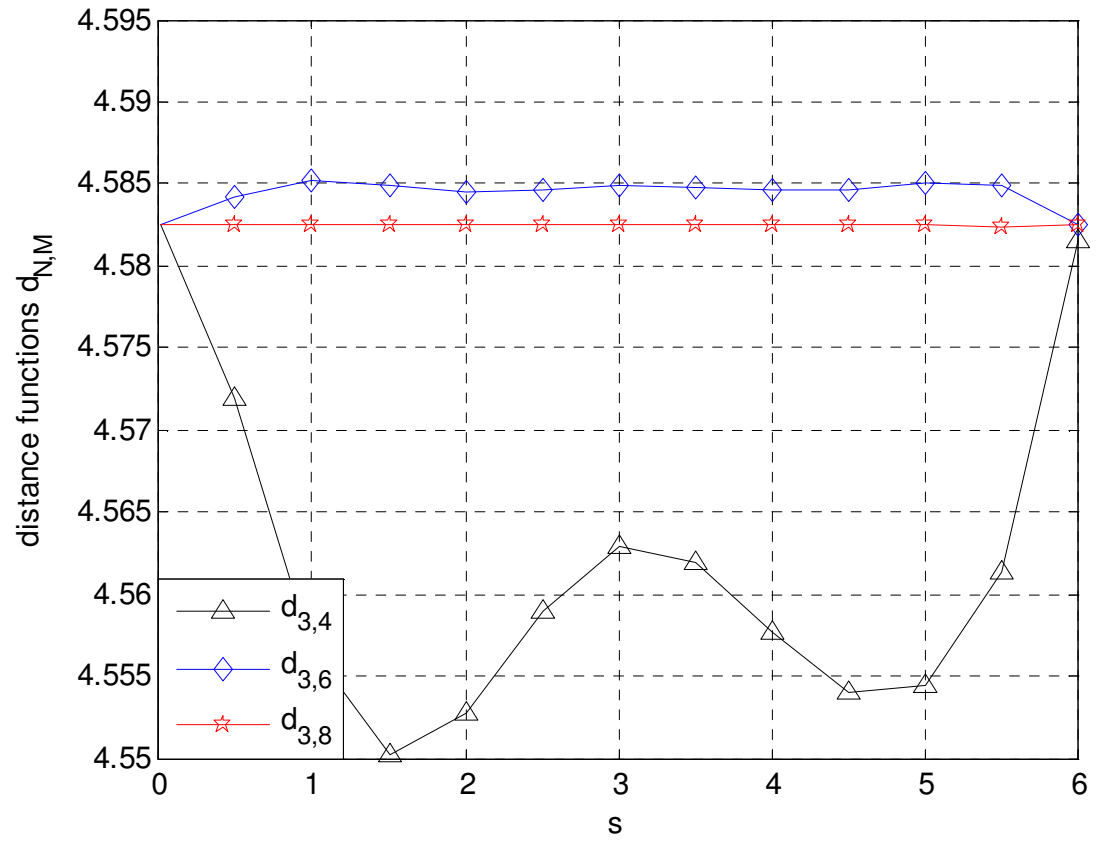

Figure 1. Comparison of distance functions

It is seen from Table 4 and Figure 1 that accuracy of the solution of system (28) increase when the value of $M$ is increased. In addition, $d_{N, M}$ is closing a constant value as the value of $M$ is selected big. This value is breadth of the curve pair of constant breadth. Hence, we can say that the present method is very effective.

Moreover, let us calculate and compare the estimated absolute error functions $\left|e_{i, N, M}(s)\right|$ for $N=3$ and 
$M=4,6,8,(i=1,2,3)$.

$$
\begin{aligned}
e_{1,3,4}(s)= & (0.6879998068 e-1) s^{2}-(0.3101720095 e-1) s^{3}+(0.358834023138630138 e-2) s^{4} \\
e_{1,3,6}(s)= & (0.5414259050 e-1) s^{2}-(0.1419290993 e-1) s^{3}+(0.780946093192976150 e-3) s^{5} \\
& -(0.328376246375317054 e-4)^{6}-(0.22724979443498158924 e-2) s^{4} \\
e_{1,3,8}(s)= & (0.5383976860 e-1) s^{2}-(0.1474210573 e-1) s^{3}-(0.449362456550112302 e-5) s^{7} \\
& +(0.139070836974095252 e-6) s^{8}-(0.16320200368388819474 e-2) s^{4} \\
& +(0.51987164532193863786 e-3) s^{5}+(0.17566163126409611616 e-4) s^{6} .
\end{aligned}
$$

Table 5: Comparison of the estimated absolute error functions $\left|e_{1, N, M}(s)\right|$ for $N=3$ and $M=4,6,8$.

\begin{tabular}{c|c|c|c}
\hline$s_{i}$ & $\left|e_{1,3,4}\left(s_{i}\right)\right|$ & $\left|e_{1,3,6}\left(s_{i}\right)\right|$ & $\left|e_{1,3,8}\left(s_{i}\right)\right|$ \\
\hline 0 & 0 & 0 & 0 \\
$\pi / 4$ & 0.0288 & 0.0259 & 0.0256 \\
$2 \pi / 4$ & 0.0714 & 0.0717 & 0.0709 \\
$3 \pi / 4$ & 0.0868 & 0.0959 & 0.0948 \\
$4 \pi / 4$ & 0.0668 & 0.0804 & 0.0790 \\
$5 \pi / 4$ & 0.0360 & 0.0439 & 0.0425 \\
$6 \pi / 4$ & 0.0515 & 0.0516 & 0.0505 \\
$7 \pi / 4$ & 0.2035 & 0.2176 & 0.2167 \\
$2 \pi$ & 0.6149 & 0.7022 & 0.6975 \\
\hline
\end{tabular}

$$
\begin{aligned}
e_{2,3,4}(s)= & (0.509072205 e-1) s^{2}-(0.2593170193 e-1) s^{3}+(0.329254069115442410 e-2) s^{4} \\
e_{2,3,6}(s)= & (0.430535746 e-1) s^{2}-(0.5323918129 e-1) s^{3}+(0.20829016919357967950 e-1) s^{4} \\
& -(0.300128817280402279 e-2) s^{5}+(0.121540192683128125 e-3) s^{6} \\
e_{2,3,8}(s)= & -(0.270211100 e-1) s^{2}+(0.2889273980 e-1) s^{3}-(0.702607695411888776 e-4) s^{7} \\
& +(0.221094199053020487 e-5) s^{8}-(0.55555395786277287546 e-2) s^{4} \\
& -(0.19018036742970799332 e-2) s^{5}+(0.71348969074471050696 e-3) s^{6} .
\end{aligned}
$$

Table 6: Comparison of the estimated absolute error functions $\left|e_{2, N, M}(s)\right|$ for $N=3$ and $M=4,6,8$.

\begin{tabular}{c|c|c|c}
\hline$s_{i}$ & $\left|e_{2,3,4}\left(s_{i}\right)\right|$ & $\left|e_{2,3,6}\left(s_{i}\right)\right|$ & $\left|e_{2,3,8}\left(s_{i}\right)\right|$ \\
\hline 0 & 0 & 0 & 0 \\
$\pi / 4$ & 0.0201 & 0.0078 & 0.0052 \\
$2 \pi / 4$ & 0.0452 & 0.0002 & 0.0024 \\
$3 \pi / 4$ & 0.0449 & 0.0126 & 0.0144 \\
$4 \pi / 4$ & 0.0191 & 0.0015 & 0.0007 \\
$5 \pi / 4$ & 0.0023 & 0.0361 & 0.0344 \\
$6 \pi / 4$ & 0.0405 & 0.0127 & 0.0105 \\
$7 \pi / 4$ & 0.2376 & 0.2349 & 0.2377 \\
$2 \pi$ & 0.7089 & 0.9556 & 0.9260 \\
\hline
\end{tabular}




$$
\begin{aligned}
e_{3,3,4}(s)= & (0.4 e-18) s+(0.1214120804) s^{2}-(0.5878895125 e-1) s^{3}+(0.694771346566892910 e-2) s^{4} \\
e_{3,3,6}(s)= & -(0.20840 e-15) s-0.1217448485 s^{2}+(0.5055487825 e-1) s^{3}+(0.453694473233072774 e-3) s^{4} \\
& -(0.205140773861414456 e-2) s^{5}+(0.179925190950981802 e-3) s^{6} \\
e_{3,3,8}(s)= & -(0.10189678 e-14) s+0.1176575974 s^{2}-(0.4562781861 e-1) s^{3} \\
& -(0.394613814952812954 e-4) s^{7}+(0.262930012001328484 e-5) s^{8}-(0.22358445035079499478 e-2) s^{4} \\
& +(0.19778385702964519922 e-2) s^{5}+(0.471203000543214462 e-5) s^{6} .
\end{aligned}
$$

Table 7: Comparison of the estimated absolute error functions $\left|e_{3, N, M}(s)\right|$ for $N=3$ and $M=4,6,8$.

\begin{tabular}{c|c|c|c}
\hline$s_{i}$ & $\left|e_{3,3,4}\left(s_{i}\right)\right|$ & $\left|e_{3,3,6}\left(s_{i}\right)\right|$ & $\left|e_{3,3,8}\left(s_{i}\right)\right|$ \\
\hline 0 & 0 & 0 & 0 \\
$\pi / 4$ & 0.0491 & 0.0510 & 0.0502 \\
$2 \pi / 4$ & 0.1140 & 0.1186 & 0.1180 \\
$3 \pi / 4$ & 0.1192 & 0.1188 & 0.1185 \\
$4 \pi / 4$ & 0.0522 & 0.0447 & 0.0442 \\
$5 \pi / 4$ & 0.0356 & 0.0361 & 0.0357 \\
$6 \pi / 4$ & 0.0297 & 0.0138 & 0.0138 \\
$7 \pi / 4$ & 0.2479 & 0.1996 & 0.1994 \\
$2 \pi$ & 1.0389 & 0.5771 & 0.6315 \\
\hline
\end{tabular}

It is seen from Table 5-6-7 that the results are almost identical and close to zero. In addition, we say that the Lucas collocation method is very effective for solving differential equations with variable coefficients. Because, It is very difficult to find the analytical solutions of these differential equations systems.

\section{Conclusions}

In this study, we have developed a new collocation method based on Lucas polynomials for solving linear differential equation system in normal form with the help of the residual error function. Then, we have given approximate solutions of system of differential equations characterizing curve pair of constant breadth by using Lucas collocation method. We have given an example to demonstrate efficiency and applicability of the present method.

In Figure 1, we have obtained the graphics of the distance function. Also, we have studied the residual error analysis. It is seen from these comparisons that the approximate solutions are very close to absolute solutions when the values of $N$ and $M$ are selected big. In addition, Lucas collacation method used for approximate solutions is very effective.

\section{References}

[1] L. Euler, De curvis triangularibus, Acta Acad. Petropol., 3-30, (1778), (1780).

[2] N. H. Ball, On ovals, American Mathematical Monthly, 37(7): 348-353,1930.

[3] E. Barbier, Note sur le probléme de I'aiguille et le jeu du joint couvert, Journal de mathématiques pures et appliquées, 2(5): 273-286, 1860. 
[4] W. Blaschke, Leibziger Berichte, 67 : 290, 1917.

[5] A. P. Mellish, Notes on Differential Geometry, Annals of Mathematics, 32(1): 181-190, 1931.

[6] M. Fujiwara, On Space Curves of Constant Breadth, Tohoku Mathematical Journal, 5: 180-184, 1914.

[7] P. C. Hammer, Constant Breadth Curves in the Plane, Procedings of the American Mathematical Society, 6(2): 333-334, 1955.

[8] F. Reuleaux, The kinematics of machinery, Trans. by A. B. W. Kennedy, Dover Pub. New York, 1963.

[9] S. Smakal, Curves of Constant Breadth, Czechoslovak Mathematical Journal, 23(1): 86-94, 1973.

[10] Ö. Köse, Düzlemde Ovaller ve Sabit Genişlikli Ĕ̆rilerin Bazı Özellikleri, Doğa Bilim Dergisi, Seri B, 8(2): 119-126, 1984.

[11] Ö. Köse, On Space Curves of Constant Breadth, Doğa Tr. J. Math, 10(1): 11-14, 1986.

[12] A. Mağden, Ö. Köse, On the Curves of Constant Breadth in E $E^{4}$-Space, Tr. J. of Mathematics, 21: 277-284, 1997.

[13] Z. Akdoğan, A. Mağden, Some Characterization of Curves of Constant Breadth in $E^{n}$-Space, Turk J Math, 25: 433-444, 2001.

[14] M. Sezer, Differential Equations Characterizing Space Curves of Constant Breadth and a Criterion for These Curves, Turkish J. of Math, 13(2): 70-78, 1989.

[15] M. Önder, H. Kocayiğit, E. Candan, Differential Equations Characterizing Timelike and Spacelike Curves of Constant Breadth in Minkowski 3-Space $E_{1}^{3}$, J. Korean Math. Soc. 48(4): 849-866, 2011.

[16] H. Kocayiğit, M. Önder, Space Curves of Constant Breadth in Minkowski 3-Space, Annali di Matematica, 192(5): 805-814, 2013.

[17] H. Kocayiğit, M. Çetin, Space Curves of Constant Breadth according to Bishop Frame in Euclidean 3-Space, New Trends in Mathematical Sciences, 2(3): 199-205, 2014.

[18] M. Çetin, M. Sezer, H. Kocayiğit, Determination of the Curves of Constant Breadth according to Bishop Frame in Euclidean 3-Space, New Trends in Mathematical Sciences (In Press).

[19] M. Sezer, A. Karamete, M. Gülsu, Taylor polynomial solutions of systems of linear differential equations with variable coefficients, International Journal of Computer Mathematics, 82(6) : 755-764, 2005.

[20] M. Çetin, M. Sezer, C. Güler, Lucas Polynomial Approach for System of High-Order Linear Differential Equations and Residual Error Estimation, Mathematical Problems in Engineering, Vol. 2015, 14 Pages, 2015.

[21] A. J. Hanson, H. Ma, Parallel transport approach to curve framing, Indiana University, Techreports-TR425, January 11, 1995.

[22] R. L. Bishop, There is More Than One Way to Frame a Curve, American Mathematical Monthly, 82(3): 246-251, 1975.

[23] A. J. Hanson, H. Ma, Quaternion Frame Approach to Streamline Visualization, IEEE Transactions on Visulation and Computer Graphics, 1(2): 164-174, 1995.

[24] F. A. Oliveira, Collocation and residual correction, Numer. Math., 36: 27-31, 1980.

[25] İ. Çelik, Approximate calculation of eigenvalues with the method of weighted residuals-collocation method, Applied Mathematics and Computation, 160(2): 401-410, 2005.

[26] S. Shahmorad, Numerical solution of the general form linear Fredholm-Volterra integro-differential equations by the Tau method with an error estimation, Applied Mathematics and Computation, 167(2): 1418-1429, 2005.

[27] İ. Çelik, Collocation method and residual correction using Chebyshev series, Applied Mathematics and Computation, 174(2): 910-920, 2006.

[28] P. Filipponi, A. F. Horadam, Second derivative sequences of Fibonacci and Lucas polynomials, The Fibonacci Quarterly, 31(3) (1993), 194-204.

[29] A. Constandache, A. Das, F. Toppan, Lucas polynomials and a standart Lax representation for the polyropic gas dynamics, Letters in Mathematical Physics, 60(3) (2002), 197-209. 